\title{
Effects of Equol Supplement on Bone and Cardiovascular Parameters in Middle-Aged Japanese Women: A Prospective Observational Study
}

\author{
Remi Yoshikata, MD, PhD, ${ }^{1,2}$ Khin Zay Yar Myint, MBBS, MHS, and Hiroaki Ohta, MD, PhD ${ }^{3}$
}

\begin{abstract}
Objective: To examine changes in the bone and cardiovascular parameters and tolerability in middle-aged Japanese women taking equol supplement for a year.

Design: This was a prospective observational study.

Subjects and Setting: Participants were 74 women receiving outpatient care at Hamasite Medical Clinic, Minato-ku, Tokyo, from 2013 to 2015.

Interventions: Participants received per oral equol-containing supplement, $10 \mathrm{mg} /$ day.

Outcome measures: The primary outcome measures were percent changes in bone and cardiovascular parameters after 1 year supplementation with equol. The secondary measures included factors affecting the parameter changes and adverse effects associated with equol use for a year.

Results: Reduction in arterial stiffness was observed after 12 months of equol supplement $(1402.3 \mathrm{~cm} / \mathrm{s}$ vs. $1367.3 \mathrm{~cm} / \mathrm{s}, p<0.001)$. Significant reductions in respective parameters were observed in women with moderate and high risk for arteriosclerosis (median [95\% confidence interval]: $-3.2 \%$ [ -5.79 to -0.74$] ;-12.65 \%$ [ -18.52 to -4.28$]$; respectively); hypertriglyceridemia $-45.53 \%$ [ -70.24 to -5.58$]$; bone resorption risk ( $-15.15 \%$ [ -23.71 to 1.56$]$; and bone fracture risk $-26.68 \%$ [ -76.43 to -5.99$]$. All 15 women with high baseline parathyroid hormone levels had achieved a median of 50\% [-54.11 to -31.69] reduction from their baseline values. These associations were further confirmed in the results of multiple linear regression analysis. There were no reported adverse events or abnormal findings in the blood chemistry, Pap smear, mammography, and transvaginal ultrasound during periodic follow-ups.

Conclusion: One year equol supplement was tolerable and induced improvement of certain bone and cardiovascular parameters, especially in higher risk groups. Further controlled studies are needed to explore longterm equol use for wellbeing of middle-aged women.
\end{abstract}

Keywords: equol, supplement, Japanese women, bone parameters, cardiovascular parameters

\section{Introduction}

$\mathbf{E}$ QUOL IS ONE of the active metabolites produced by the action of colonic bacteria on soy isoflavones. Dietary isoflavones are mainly made up of glycoside forms such as glycitin, daizin, and genistein, which are not readily absorbed across the enterocytes owing to their large hydrophilic struc- tures. ${ }^{1,2}$ In the lumen of the small and large intestine, sugar moieties from these glycosides are hydrolyzed to form aglycones (glycitein, daidzein, and genistein) by $\beta$-glycosidases in the small intestinal brush border or colonic bacteria. ${ }^{3,4}$ Some aglycones are absorbed after being hydrolyzed and some remain unabsorbed in the intestinal lumen and are converted to final metabolites, such as equol and O-desmethylangolensin,

\footnotetext{
${ }^{1}$ Hamasite Clinic, Minato-ku, Japan.

${ }^{2}$ Tokyo Midtown Medical Center, Minato-ku, Japan.

${ }^{3}$ Sanno Medical Center, Minato-ku, Japan.
}

(c) Remi Yoshikata et al., 2018; Published by Mary Ann Liebert, Inc. This Open Access article is distributed under the terms of the Creative Commons License (http://creativecommons.org/licenses/by/4.0), which permits the unrestricted use, distribution, and reproduction in any medium, provided the original work is properly cited. 
by the action of colonic bacteria. ${ }^{5,6}$ These final metabolites are absorbed and transported into the liver via the hepatic portal circulation.

The bioavailabilities and biological activities of isoflavones are influenced by the type of diet and the ability of host colonic bacteria to metabolize them into final active metabolites. ${ }^{7}$ As for the metabolism of equol, certain bacteria species are required for the production of equol from daidzein, ${ }^{8}$ and a host genetic variant might be one of the determinants for variations in equol metabolism. ${ }^{9}$ Therefore, there is a great variation in the individual ability to produce equol. Although $50 \%$ of Japanese women are reported to have this equol-producing ability, only about $30 \%$ of Japanese women were found to be equol producers in our recent study. ${ }^{10}$ Changes in diet and the widespread use of antibiotics might have led to the reduction in the number of people who are equol producers. ${ }^{11,12}$

Equol resembles the chemical structure of the female hormone estrogen and it is thought to exert estrogenic actions by genomic pathways through estrogen receptors $\alpha$ and $\beta$ in the cytoplasm and nucleus ${ }^{13,14}$ and by a rapid nongenomic pathway through $G$ protein-coupled estrogen receptors. ${ }^{15,16}$ There is increasing evidence of its efficacy in relieving climacteric symptoms, ${ }^{17,18}$ and antiatherosclerotic actions. ${ }^{19}$ In addition, its potential action in slowing bone mineral density (BMD) loss, ${ }^{20,21}$ prevention of hormonedependent cancers such as breast cancer ${ }^{22,23}$ and prostate ${ }^{24,25}$ cancers in addition to protecting against aging, diabetes, and obesity have been investigated. ${ }^{26,27}$

The efficacy of soy is greatly dependent on the host's ability to convert equol in the intestine by microflora. In our previous study, vascular and bone biomarkers showed favorable trends for women around the menopausal age who had the ability to produce equol. ${ }^{10}$ Therefore, it is postulated that the same age group of women might also benefit from dietary equol supplement. Clinical studies on equol supplements have focused on menopausal symptoms, lipid and metabolic biomarkers, and arterial stiffness, ${ }^{17,28-30}$ suggesting that equol supplementation has some positive effects. These studies have focused on equol's efficacy in nonproducer population. Furthermore, they are mostly short-term clinical trials and there is a need to evaluate its long-term efficacy and safety.

In this study, our primary objective was to examine the changes in bone and cardiovascular parameters among women taking equol supplement for a year. Our secondary objectives were to find out the factors affecting the parameter changes such as equol-producer status, and adverse effects associated with equol use for a year.

\section{Materials and Methods}

\section{Study population}

This prospective observational study was conducted among 105 women who were receiving outpatient care at the Hamasite Medical Clinic from 2013 to 2015 and who gave their consent to participate in the study. Among them, 74 women (44-74 years of age) completed the 12 month follow-up for blood and urine testing for bone and cardiovascular parameters (Fig. 1). Approval for the study was obtained from the Institutional Review Board of Tokyo Midtown Medical Corporation. The background characteristics of the participants were described in Table 1. Menopause was defined as no menstruation at least 12 months since last menstrual pe- riod or bilateral oophorectomy. Lifestyle-related diseases included hypertension, dyslipidemia, and diabetes. According to Japan Osteoporosis Society, osteoporosis was defined as having BMD of $70 \%$ and below in the absence of fragility fracture, or having BMD between $70 \%$ and $80 \%$ in the presence of fragility fracture, compared to average BMD values in young healthy women (Young Adult Mean: YAM).

\section{Study treatments}

Participants received per oral equol-containing supplement, $10 \mathrm{mg} /$ day. Among them, 22 women received combined hormone replacement therapy (HRT) as transdermal patch every 2 weeks. Decisions on equol supplement alone or combination with HRT were based on clinically evaluated facts including their most troubling symptoms, background medical history, and willingness to receive equol only or both therapies. Each equol capsule contained $98 \%$ S-equol, $2 \%$ daidzein, $0.2 \%$ glycitein, and $0.1 \%$ genistein extracted from fermented soy beans (product name: FlavoCel EQ-5; Daicel Corporation, Tokyo, Japan). One transdermal combined HRT patch contained estradiol $0.62 \mathrm{mg}$ and norethistrone acetate $2.7 \mathrm{mg}$ (product name: Menoaid Combipatch; Aska Pharmaceutical Co., Ltd., Tokyo, Japan). Treatments for pre-existing diseases such as lifestylerelated diseases or osteoporosis were allowed to continue.

\section{Determination of equol-producer status at baseline}

Early morning urine samples were collected and urinary equol was measured by Healthcare Systems Co., Ltd. using Soy Check, which is an immunochromatographic strip test. The measurement method was as described in our previous study. ${ }^{10}$ Participants were defined as equol producers with a urinary equol level higher than $1.0 \mu \mathrm{mol} / \mathrm{L}$. ${ }^{3,32}$

\section{Measurement of parameters}

Anthropometric measures such as body height and weight were measured using a height weight scale (A \& D Company Limited, Tokyo, Japan). Blood samples were obtained after an overnight fast to determine levels of triglycerides (TG), total cholesterol, low density lipoprotein (LDL) cholesterol, high density lipoprotein (HDL) cholesterol, intact parathyroid hormone (PTH), calcium, phosphorous, and procollagen type I propeptide (P1NP). Urinary calcium and N-telopeptide (NTX) levels were measured using early morning urine samples. To assess the degree of arterial stiffness (arteriosclerosis), branchial-ankle pulse wave velocity (baPWV) was measured using vascular ultrasound (Fukuda Denshi, Tokyo, Japan). These measurements were done at baseline and at 3 monthly follow-ups for baPWV and 6-monthly follow-ups for other parameters up to 12 months.

\section{Monitoring of adverse effects}

Clinical signs and symptoms were monitored during monthly follow-ups. Liver and kidney function tests, and complete blood counts were done every 6 months. Pap smear, transvaginal ultrasound, and mammography were performed at baseline and after 12 months.

\section{Statistical analyses}

Statistical analyses were performed using IBM SPSS Statistics version 19 software (IBM Japan, Minato-ku, 


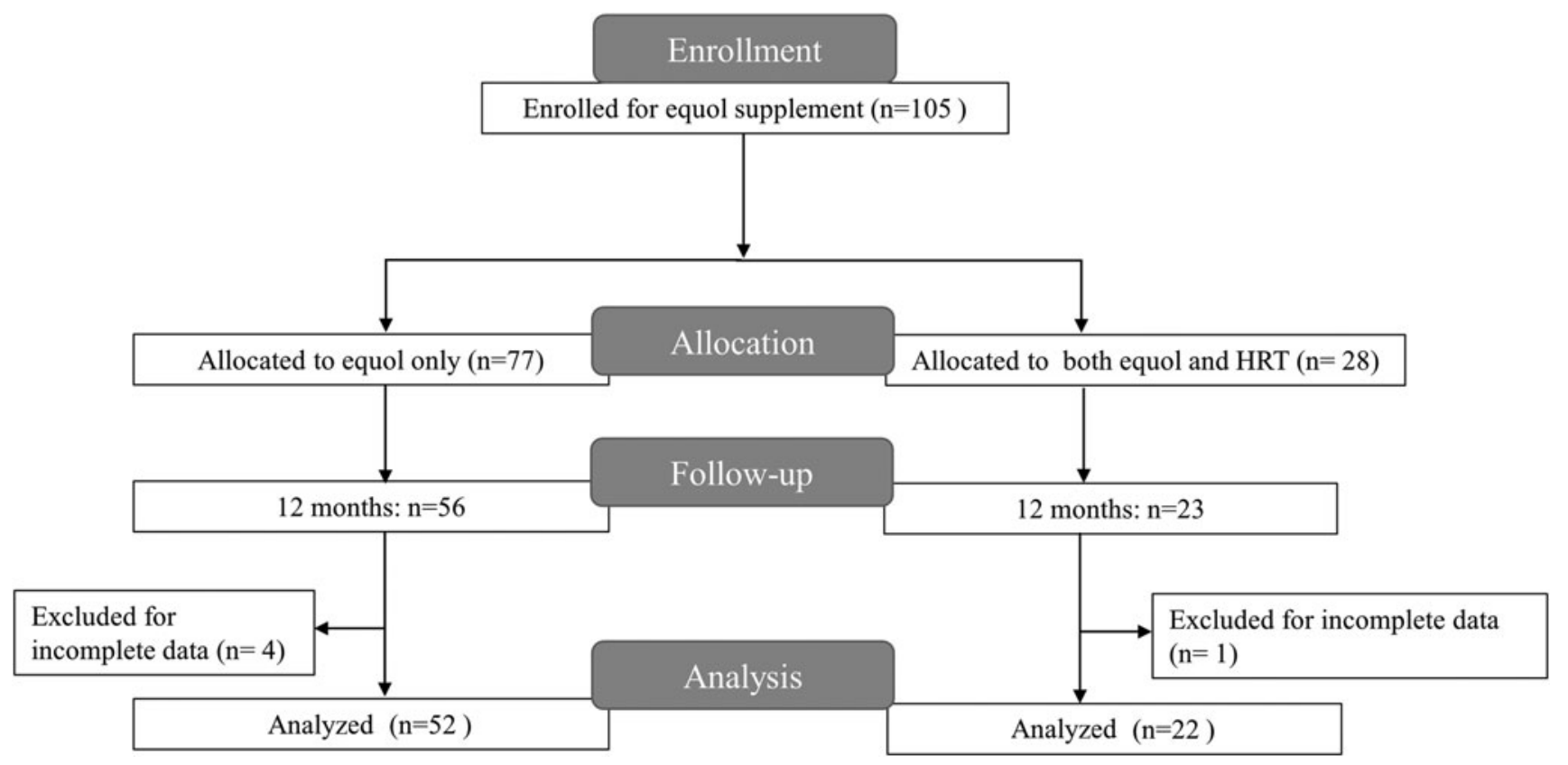

FIG. 1. Flow chart of the observational study. HRT, hormone replacement therapy.

Tokyo, Japan). Distribution of normality was assessed with the Kolmogorov-Smirnov test, box plots, and histograms. Categorical variables were expressed as numbers $(N)$ and percentages $(\%)$. Continuous variables were expressed as means or medians. Baseline parameters between equol producers and nonproducers were compared using MannWhitney tests. Pre- and post-parameters were compared using Wilcoxon signed-rank tests. Percent changes of parameter values between the baseline and at 12 months were calculated and compared among different initial risk groups. These initial risk groups were categorized according to reference standards in Japan.

Multiple linear regression analyses were further conducted to confirm the associations between the percent changes in the parameters and their respective initial risk categories. The models were adjusted for age, equol-producing phenotype, HRT treatment; and treatments for osteoporosis in the analysis for bone parameters and lifestyle-related diseases for analysis of cardiovascular parameters. All tests were twosided, and statistical significance was defined as $p<0.05$.

Table 1. Basic Characteristics of the Participants

Total $(\mathrm{N}=74)$

Age, years $\pm \mathrm{SD}$

$55.14( \pm 7.8)$

Age at menopause, years $\pm \mathrm{SD}$

BMI, $\mathrm{kg} / \mathrm{m}^{2}$

Before menopause/menopause, $N$

Equol producers, $n(\%)$

HRT treatment, $n(\%)$

Lifestyle-related diseases and treatment, $n(\%)$

Osteoporosis and treatment, $n(\%)$

$49.33( \pm 4.7)$

$21.6( \pm 3.4)$

$30 / 44$

$25^{\mathrm{a}}(33.8 \%)$

$22(29.7 \%)$

$31(41.9 \%)$

$21(28.4 \%)$

Categorical values are shown as numbers and proportions (\%) and continuous values are shown as mean ( \pm standard deviation).

${ }^{a}$ Equol-producer status was unknown for two participants.

BMI, Body-mass index; HRT, hormone replacement therapy.

\section{Results}

Equol producers $(n=25)$ had a lower baseline baPWV (a measure of arterial stiffness) compared to nonproducers $(n=47)$, but the results has missed statistical significance $(972 \mathrm{~cm} / \mathrm{s}$ vs. $1656 \mathrm{~cm} / \mathrm{s}, p=0.482)$. Similarly, there were no significant differences between equol producers and nonproducers in lipid and bone parameters at the baseline. Significant reduction in baPWV, was observed as early as 3 months after equol supplementation in the group of women taking only equol supplement who showed up for 3-month follow-up $(1429 \mathrm{~cm} / \mathrm{s}$ vs. $1311 \mathrm{~cm} / \mathrm{s}, p<0.001, n=45)$. In women who had completed 12-month follow-up, there was a significant reduction in arterial stiffness, increased levels of total cholesterol, LDL and HDL cholesterol. However, LDL/HDL ratios showed no significant change. Bone parameters also showed decreasing trends, however, they were not statistically significant (Fig. 2).

As shown in Figure 3, significant reductions in baPWV were noted in patients with moderate arteriosclerotic risk (baPWV $1400-1800 \mathrm{~cm} / \mathrm{s}, n=30$ ) and in high arteriosclerotic risk (baPWV $\geqq 1800 \mathrm{~cm} / \mathrm{s}, n=8$ ). In addition, significant reductions in parameters were observed in patients with hypertriglyceridemia ( $\mathrm{TG} \geqq 150 \mathrm{mg} / \mathrm{dL}, n=6$ ), those with high risk for bone resorption (NTX 35.3-54.3 nmol/L BCE/mM.Cre, $n=32$ ) or fracture (NTX $>54.3 \mathrm{nmol} / \mathrm{L} \mathrm{BCE} / \mathrm{mM}$.Cre, $n=5$ ), and all the women with abnormal parathyroid hormone levels (PTH $>65 \mathrm{pg} / \mathrm{mL}, n=15$ ) at the baseline.

As shown in Table 2, these initial risk categories were still the main predictors of percent decline in the parameter levels, except for baPWV, in the multiple regression analyses. In addition, there was a negative association with the participant's age and percent change in TG level after 12 months. In other words, younger women could be benefitted from reduction in TG levels after 12 months. Equol producers had a tendency to have reduced urinary NTX levels compared to equol nonproducers; however, this association missed a statistical significance. 


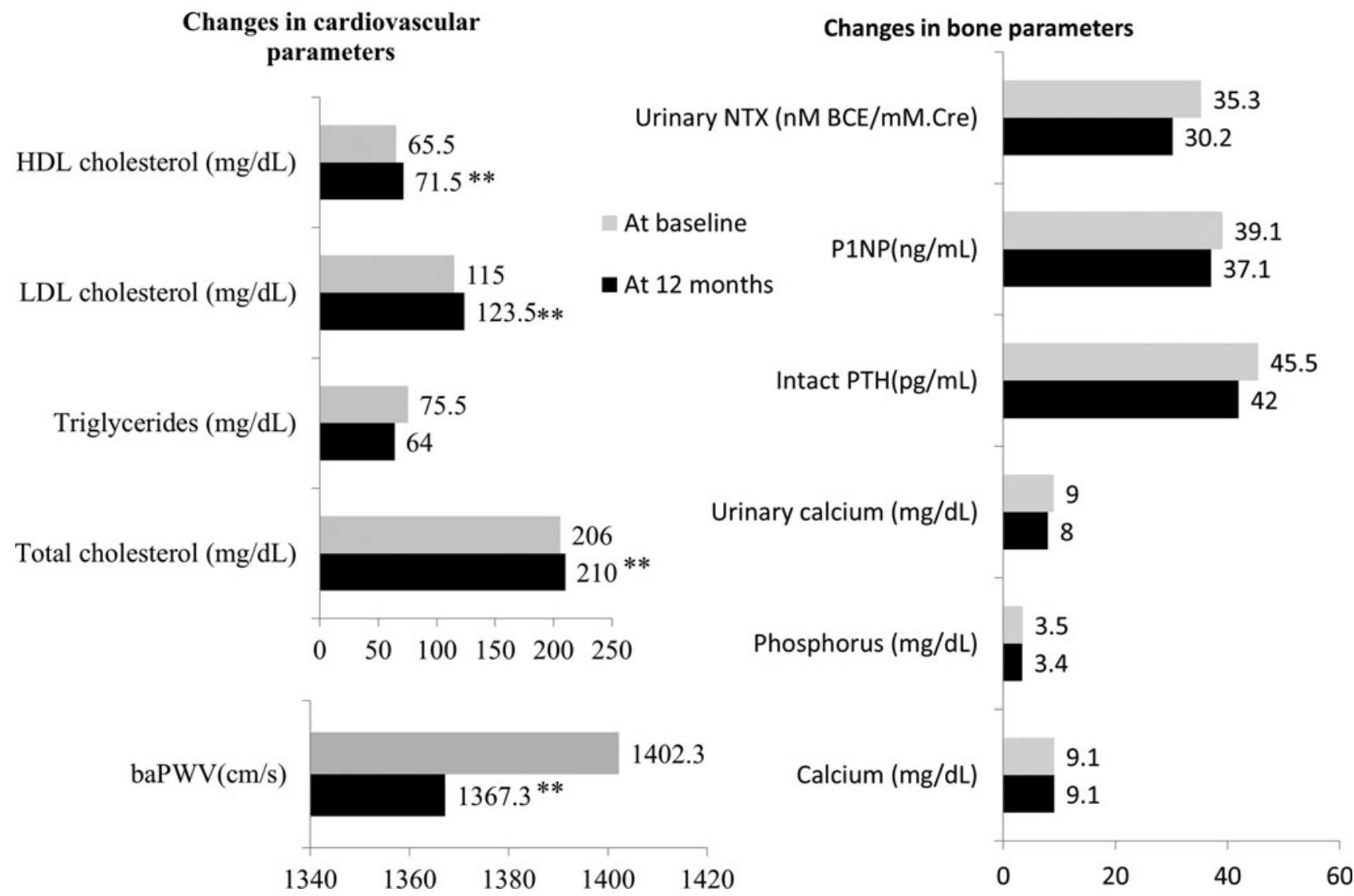

FIG. 2. Comparison of parameters at baseline and 12 months after equol supplement $(\mathrm{n}=74)$. LDL, HDL and total cholesterol levels were significantly increased. Arterial stiffness was improved significantly. Bone parameters showed a declining trend, which is not statistically significant. Values at baseline and 12th month post-treatment are shown as medians. $p$-Values are assessed by Wilcoxon signed rank test and statistically significant differences $(* * p<0.01)$. baPWV, branchial-ankle pulse wave velocity; HDL, high density lipoprotein; LDL, low density lipoprotein cholesterol; NTX, Ntelopeptide; P1NP, procollagen type I propeptide; PTH, parathyroid hormone.

\section{Safety}

There were no reported adverse events or abnormal findings in blood cells, liver and kidney function tests, at periodic follow-ups. Transvaginal ultrasound for endometrial thickness, Pap smear, and mammography showed no significant changes after 12 months. In addition, no significant changes were noted in underlying gynecological conditions such as uterine fibroids.

\section{Discussion}

Equol-producer status has been associated with decreased blood pressure and improved arterial stiffness and endothelial function ${ }^{30,33,34}$ Being an equol producer has been associated with reduced risk for coronary calcification in Japanese male adults. ${ }^{35}$ We have observed favorable cardiovascular effects such as reduced baPWV, homocysteine, high-sensitivity $\mathrm{C}$-reactive protein, and high eicosapentanoic acid to arachidonic acid ratio in equol-producing women around 50 and 60 years of age. ${ }^{10}$ The baseline median baPWV value was lower in the equol producer group in this study but it was not statistically significant, which is contrary to our previous study. The reason for this discrepancy might be explained by being a small sample size and dif- ferent target population. Our previous study found the association of equol-producer status with reduced baPWV among 214 women in the regular medical check-up who were relatively healthy. On the other hand, 74 women in this study came to the clinic for postmenopausal or certain medical complaints, where naturally occurring equol might be insufficient for body homeostasis. The same reason could be considered for nonsignificant results between equol producers and nonproducers in lipid and bone parameters at the baseline.

The effect of equol supplementation on arterial stiffness was reported in some short-term clinical studies. ${ }^{28,30}$ Equol significantly induced reduction in baPWV as early as 3 months after supplementation, compared with HRT, in our unpublished small clinical study.* This is the first study in which a significant reduction in arterial stiffness was

\footnotetext{
*Yoshikata R, Ohta H, Myint K. Comparison of equol containing dietary supplement with hormone replacement therapy in the improvement of vasomotor symptoms and secondary vascular actions in women with climacteric symptoms. Poster presented at the annual meeting of the North American Menopause Society, Las Vegas, NV, October 2015. Abs
} 

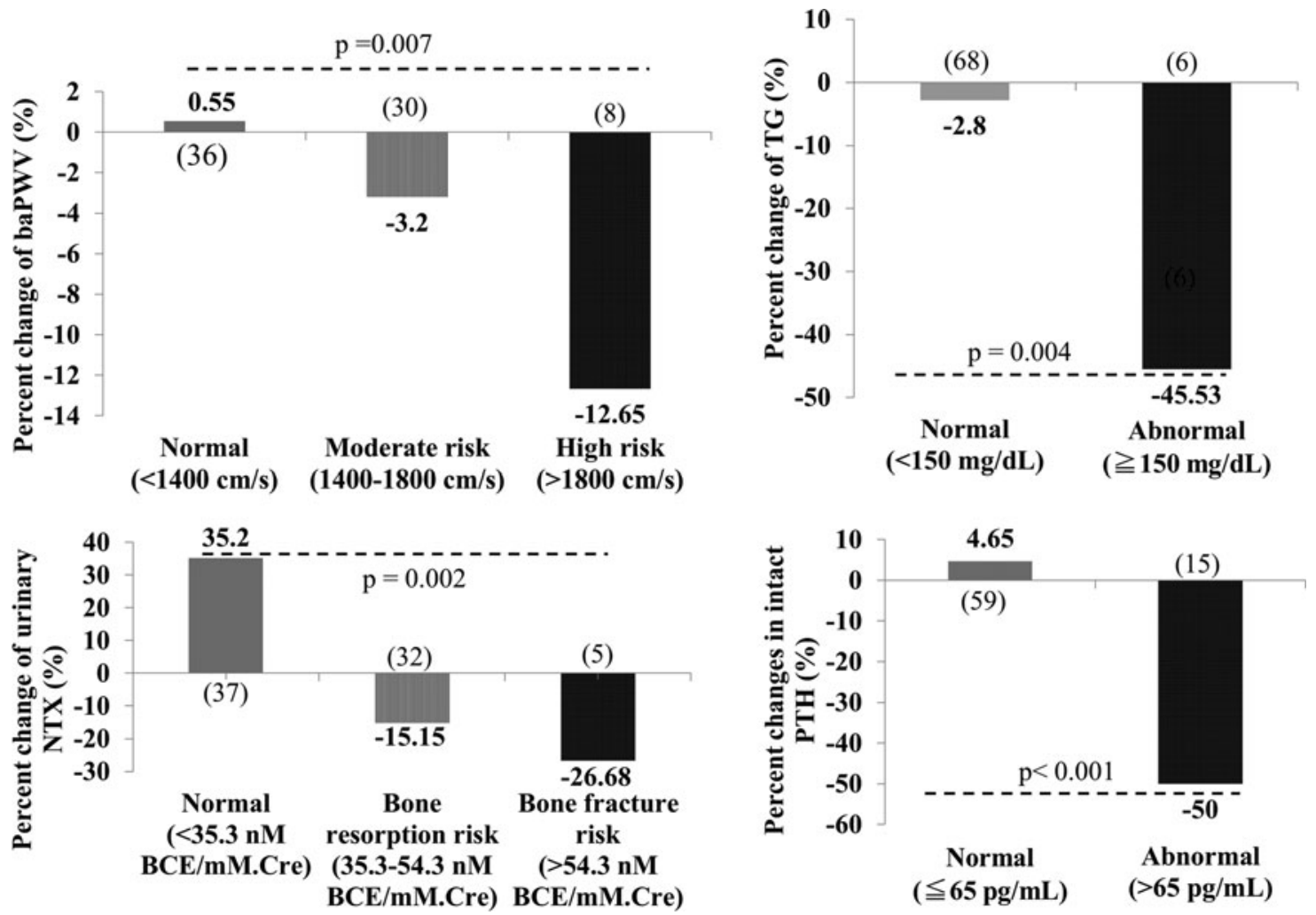

FIG. 3. Post-treatment changes in parameters with respect to their initial risk categories. People in the high risk categories showed reduction in the above parameters. The values were expressed in medians, and $p$-values were assessed by Kruskal-Wallis tests. (), number of patients; baPWV, branchial-ankle pulse wave velocity; NTX, N-telopeptide; PTH, parathyroid hormone; TG, triglycerides.

reported after 12-month supplementation with equol. The reduction in baPWV was more prominent among those with high baseline risks for arteriosclerosis, who had baPWV more than $1400 \mathrm{~cm} / \mathrm{s}$ at the baseline. The mechanism of equol actions on arteriosclerosis might be not only via conventional estrogen receptors but also through the production of vasodilators such as nitric oxide. ${ }^{36,37}$

Regarding blood lipids, equol producers tend to have better improvements compared to nonproducers, ${ }^{10,38,39}$ and therefore, equol supplementation is expected to positively affect lipid levels. The mechanism of equol action on blood lipids might be owing to increased insulin sensitivity through estrogen receptor beta $(E R \beta)$-mediated regulation of lipogenesis and glucose metabolism, similar to intrinsic estrogen. ${ }^{27,39}$ In this study, we observed that good cholesterol (HDL) levels were increased after equol treatment; however, the bad cholesterol level had also increased and the index for arteriosclerosis (LDL/HDL ratio) showed no significant change. These results were hard to discern and further well-designed clinical trials are warranted to examine the effects of equol on blood cholesterol level. It is also interesting that, although the decline in TG levels was not significant in all participants, women with hypertriglyceridemia $(>150 \mathrm{mg} / \mathrm{dL})$ were more likely to benefit from equol treatment, even after multivariate analysis including medication history.

Menopause is said to be associated with a rapid phase of bone loss, resulting from an imbalance between bone resorption and bone formation due to estrogen deficiency. ${ }^{40}$ Women who are equol producers showed slower bone density reduction compared with equol nonproducers ${ }^{41,42}$ and there was association of equol-producer status and reduction of bone resorption markers, such as urinary deoxypyridinoline $^{43}$ and urinary NTX. ${ }^{10}$ Studies using animal cell lines have shown that equol inhibits osteoclast formation, ${ }^{44}$ a result that may be attributed to reduction in bone resorption markers. In this study, we found that women with the high baseline risk for bone resorption or fracture were more likely to have reduced urinary NTX levels after equol intervention; this is the first such finding among women in a clinical setting.

PTH levels were greatly decreased after the intervention in 15 women with abnormal PTH levels $($ PTH $>65 \mathrm{pg} / \mathrm{mL})$. To be exact, all those women achieved normal PTH levels, that is, below $65 \mathrm{pg} / \mathrm{mL}$, after 12 months supplementation with equol. There are conflicting reports on the presence of estrogen receptors in parathyroid glands. However, the regulation of PTH is mediated mostly by fibroblast growth factor 23 (FGF23) ${ }^{45}$ and its expression has been shown to be under the influence of estrogen in rats. ${ }^{46}$ Additionally, estrogen actions on $\mathrm{Ca}$, vitamin D, and phosphorous might indirectly affect the negative feedback regulation of $\mathrm{PTH},{ }^{47}$ and equol's estrogenic action might be responsible for reduction in intact PTH level. There have been no previous reports on the relationship between intact PTH and isoflavones; therefore, ours is the first study to report this association.

This study has several limitations. First, the change in food habits and supplement history were not assessed, and therefore, the outcomes of the study might have been 
Table 2. Summary of Multiple Regression Analyses $(N=72)$

\begin{tabular}{|c|c|c|c|c|}
\hline \multirow[b]{2}{*}{ Variable } & \multirow[b]{2}{*}{ B } & \multicolumn{2}{|c|}{$95 \% C I$} & \multirow[b]{2}{*}{$\mathrm{p}$} \\
\hline & & Lower limit & Upper limit & \\
\hline \multicolumn{5}{|c|}{ Dependent variable: percent changes in baPWV } \\
\hline Initial baPWV risk group & -5.440 & -11.374 & 0.494 & 0.072 \\
\hline Age & 0.221 & -0.268 & 0.710 & 0.371 \\
\hline HRT treatment & -4.477 & -11.187 & 2.232 & 0.187 \\
\hline Equol producer & 2.284 & -4.198 & 8.766 & 0.484 \\
\hline Lifestyle-related disease treatment & 3.618 & -2.879 & 10.116 & 0.270 \\
\hline \multicolumn{5}{|c|}{ Dependent variable: percent changes in TG } \\
\hline Initial TG risk group & -50.345 & -80.625 & -20.064 & 0.001 \\
\hline Age & 1.162 & 0.072 & 2.252 & $\mathbf{0 . 0 3 7}$ \\
\hline HRT treatment & 0.747 & -16.915 & 18.410 & 0.933 \\
\hline Equol producer & 4.799 & -12.067 & 21.664 & 0.572 \\
\hline Lifestyle-related disease treatment & 6.146 & -10.490 & 22.783 & 0.463 \\
\hline \multicolumn{5}{|c|}{ Dependent variable: percent changes in urinary NTX } \\
\hline Initial urinary NTX risk group & -45.188 & -68.337 & -22.039 & $<0.001$ \\
\hline Age & -0.635 & -2.560 & 1.290 & 0.512 \\
\hline HRT treatment & -24.134 & -54.358 & 6.090 & 0.116 \\
\hline Equol producer & -27.642 & -56.310 & 1.025 & 0.059 \\
\hline Osteoporosis treatment & -5.454 & -40.079 & 29.172 & 0.754 \\
\hline \multicolumn{5}{|c|}{ Dependent variable: percent changes in PTH } \\
\hline Initial PTH risk group & -51.691 & -75.118 & -28.265 & $<0.001$ \\
\hline Age & 0.392 & -0.912 & 1.697 & 0.550 \\
\hline HRT treatment & -3.053 & -23.557 & 17.451 & 0.767 \\
\hline Equol producer & -13.463 & -33.147 & 6.221 & 0.177 \\
\hline Osteoporosis treatment & -8.160 & -31.343 & 15.023 & 0.485 \\
\hline
\end{tabular}

$p<0.05$ were bold.

$\mathrm{B}$, unstandardized regression coefficient; baPWV, branchial-ankle pulse wave velocity; CI, confidence interval (of partial correlation coefficients); HRT, hormone replacement therapy; NTX, N-telopeptide; PTH, parathyroid hormone; TG, triglyceride.

affected by the type of diets such as isoflavones or prebiotics and other relevant food habits. ${ }^{48,49}$ However, diet is not the sole contributor for gut environment and equol metabolism or actions. For instance, host genetic variants might also be responsible for variations in equol metabolism and bioavailability. ${ }^{7,9}$ Also, it takes time to convert an equol nonproducer to a producer. Even for the producers to consistently maintain the efficacy of equol, that is, to maintain the blood level of biologically available equol, they need to consume at least $50 \mathrm{mg}$ of soy isoflavones daily, which is very difficult to achieve. In addition, women in this study were patients with postmenopausal or medical problems, who might require more potent dosage of equol rather than a trace amount of naturally occurring equol inside the body. For those reasons, we assumed that the effect of diet was modest compared to daily equol supplementation in these women.

Second, our study outcomes had mainly focused on the biomarkers rather than the patient-centered outcomes. However, with the improvements seen in baPWV (arterial stiffness), we have clinically observed that most patients enjoyed better blood pressure control and relief in vasomotor symptoms such as hot flashes along with the stable blood pressure. In fact, we also noticed significant improvements in the perceived climacteric symptoms, assessed in the monthly questionnaires. The improvements were observed in both groups taking equol alone and combination with HRT, regardless of equol-producing ability (Supplementary Data; Supplementary Data are available online at www.liebertpub.com/acm). However, those results should be taken with caution as there was no placebo group and we could not definitely exclude the improvements as a result of time.

Third, we could not confirm the $100 \%$ adherence to equol supplementation in all the women who have completed the 12-month follow-up. Only $80 \%$ of them (60 out of 74 women) returned the monthly questionnaires, noting that they had been taking the supplement almost every day. Fourth, there was no comparison with a placebo group and sample size was limited as this was a prospective, observational study. Therefore, there is a possibility that the efficacy and safety of equol supplementation might have been missed due to potential type II error. Larger confirmatory studies are needed to warrant the findings.

Despite these limitations, our study is, notably, the first to examine the effects of 1-year supplementation, especially on bone parameters, and tolerability using follow-up screening for cervical and breast cancers in addition to endometrial conditions. Since this study could provide valuable information on both efficacy and safety, it could be useful as a reference study for those who want to try equol as an alternative or complementary therapy for the wellbeing of middle-aged women in real clinical setting.

\section{Conclusion}

This study suggested that reduction in arterial stiffness was possible after 12 month supplementation with equol. Especially, positive responses could be seen in women in the high-risk groups such as those with hyperglycemia, high 
risk for arteriosclerosis, bone resorption and fracture, and abnormal PTH levels. These positive effects of equol alone or in combination with HRT suggest that equol might behave in a manner similar to selective estrogen receptor modulators. It might serve as a new, promising, and safe therapeutic option to offer as an alternative or complementary therapy in addition to proven therapies for women affected by estrogen deficiency. In addition, we observed that 1-year equol supplementation was well tolerated without any adverse events. Future well-designed randomized controlled trials are needed to validate the long-term use of equol for customized care of women, especially in those in the late reproductive life who are starting to be affected by low levels of intrinsic estrogen.

\section{Acknowledgments}

We would like to acknowledge all the women who willingly participated in the study.

\section{Author Disclosure Statement}

No competing financial interest exits.

\section{References}

1. Xu X, Harris K, Wang H, Murphy P. Bioavailability of soybean isoflavones depends upon gut microflora in women. J Nutr 1995;125:2307-2315.

2. Liu Y, Hu M. Absorption and metabolism of flavonoids in the Caco-2 cell culture model and a perused rat intestinal model. Drug Metab Dispos 2002;30:370-377.

3. Griffiths LA, Barrow A. Metabolism of flavonoid compounds in germ-free rats. Biochem J 1972;72:1161-1162.

4. Day A, Dupont M, Ridley S, et al. Deglycosylation of flavonoid and isoflavonoid glycosides by human small intestine and liver $\beta$-glucosidase activity. Febs Lett 1998;436: 71-75.

5. Atkinson C, Frankenfield CL, Lampe JW. Gut bacterial metabolism of the soy isoflavone daidzein: Exploring the relevance to human health. Exp Biol Med 2005;230:155170.

6. Decroos K, Vanhemmens S, Cattoir S, et al. Isolation and characterisation of an equol-producing mixed microbial culture from a human faecal sample and its activity under gastrointestinal conditions. Arch Microbiol 2005;183:45-55.

7. Miura A, Sugiyama C, Sakakibara H, et al. Bioavailability of isoflavones from soy products in equol producers and non-producers in Japanese women. J Nutr Intermed Metab 2016;6:41-47.

8. Rafii F. The role of colonic bacteria in the metabolism of the natural isoflavone daidzin to equol. Metabolites 2015;5: 56-73.

9. Van de Merwe JP, Stegeman JH, Hazenberg MP. The resident faecal flora is determined by genetic characteristics of the host. Implications for Crohn's disease? Antonie Van Leeuwenhoek 1983;49:119-124.

10. Yoshikata R, Myint KZ, Ohta H. Relationship between equol producer status and metabolic parameters in 743 Japanese women. Menopause 2017;24:216-222.

11. Van der Velpen V, Hollman PC, van Nielen M, et al. Large inter-individual variation in isoflavone plasma concentration limits use of isoflavone intake data for risk assessment. Eur J Clin Nutr 2014;68:1141-1147.
12. Franke AA, Lai JF, Halm BM, et al. Equol production changes over time in postmenopausal women. J Nutr Biochem 2012;23:573-579.

13. Klinge CM. Estrogen receptor interaction with estrogen response elements. Nucleic Acids Res 2001;29:2905-2919.

14. Marino M, Galluzzo P, Ascenzi P. Estrogen signaling multiple pathways to impact gene transcription. Curr Genomics 2006;7:497-508.

15. Nilsson S, Mäkelä S, Treuter E, et al. Mechanisms of estrogen action. Physiol Rev 2001;81:1535-1565.

16. Heldring N, Pike A, Andersson S, et al. Estrogen receptors: How do they signal and what are their targets. Physiol Rev 2007;87:905-931.

17. Ishiwata N, Melby MK, Mizuno S, Watanabe S. New equol supplement for relieving menopausal symptoms: Randomized, placebo-controlled trial of Japanese women. Menopause 2009;16:141-148.

18. Aso T. Equol improves menopausal symptoms in Japanese women. J Nutr 2010;140:1386S-1389S.

19. Potter SM, Baum JA, Teng H, et al. Soy protein and isoflavones: Their effects on blood lipids and bone density in postmenopausal women. Am J Clin Nutr 1998;68:1375S1379S.

20. Morabito N, Crisafulli A, Vergara C, et al. Effects of genistein and hormone-replacement therapy on bone loss in early postmenopausal women: A randomized double-blind placebo-controlled study. J Bone Miner Res 2002;17:19041912.

21. Alekel DL, Germain AS, Peterson CT, et al. Isoflavone-rich soy protein isolate attenuates bone loss in the lumbar spine of perimenopausal women. Am J Clin Nutr 2000;72:844852 .

22. Zheng W, Dai Q, Custer LJ, et al. Urinary excretion of isoflavonoids and the risk of breast cancer. Cancer Epidemiol Biomarkers Prev 1999;8:35-40.

23. Ingram D, Sanders K, Kolybaba M, Lopez D. Case-control study of phyto-oestrogens and breast cancer. Lancet 1997; 350:990-994.

24. Akaza H, Miyanaga N, Takashima N, et al. Is daidzein nonmetabolizer a high risk for prostate cancer? A case-controlled study of serum soybean isoflavone concentration. Jpn J Clin Oncol 2002;32:296-300.

25. Ozasa K, Nakao M, Watanabe Y, et al. Serum phytoestrogens and prostate cancer risk in a nested case-control study among Japanese men. Cancer Sci 2004;95:65-71.

26. Lephart ED. Skin aging and oxidative stress: Equol's antiaging effects via biochemical and molecular mechanisms. Ageing Res Rev 2016;11:36-54.

27. Mauvais-Jarvis F, Clegg DJ, Hevener AL. The role of estrogens in control of energy balance and glucose homeostasis. Endocr Rev 2013;34:309-338.

28. Usui T, Tochiya M, Sasaki Y, et al. Effects of natural Sequol supplements on overweight or obesity and metabolic syndrome in the Japanese, based on sex and equol status. Clin Endocrinol 2013;78:365-372.

29. Jenks BH, Iwashita S, Nakagawa Y, et al. A pilot study on the effects of S-equol compared to soy isoflavones on menopausal hot flash frequency. J Womens Health (Larchmt) 2012;21:674-682.

30. Hazim S, Curtis PJ, Schär MY, et al. Acute benefits of the microbial-derived isoflavone metabolite equol on arterial stiffness in men prospectively recruited according to equol producer phenotype: A double-blind randomized controlled trial. Am J Clin Nutr 2016;103:694-702. 
31. Lampe JW, Karr SC, Hutchins AM, Slavin, JL. Urinary equol excretion with a soy challenge: influence of habitual diet. Proc Soc Exp Biol Med 1998;217:335-339.

32. Rowland IR, Wiseman H, Sanders TAB, et al. Interindividual variation in metabolism of soy isoflavones and lignans: Influence of habitual diet on equol production by the gut microflora nutrition and cancer. Nutr Cancer 2000;36:27-32.

33. Tormala RM, Appt S, Clarkson TB, et al. Individual differences in equol production capability modulate blood pressure in tibolone-treated postmenopausal women: Lack of effect of soy supplementation. Climacteric 2007;10:471-479.

34. Tormala RM, Appt S, Clarkson TB, et al. Equol production capability is associated with favorable vascular function in postmenopausal women using tibolone; no effect with soy supplementation. Atherosclerosis 2008;198:174-178.

35. Ahuja V, Miura K, Vishnu A, et al. Significant inverse association of equol-producer status with coronary artery calcification but not dietary isoflavones in healthy Japanese men. Brit J Nutr 2017;117:260-266.

36. Joy S, Siow RC, Rowlands DJ, et al. The isoflavone Equol mediates rapid vascular relaxation: $\mathrm{Ca} 2+-$ independent activation of endothelial nitric-oxide synthase/Hsp90 involving ERK1/2 and Akt phosphorylation in human endothelial cells. J Biol Chem 2006;281:27335-27345.

37. Kang JS, Yoon YD, Han MH, et al. Equol inhibits nitric oxide production and inducible nitric oxide synthase gene expression through down-regulating the activation of Akt. Int Immunopharmacol 2007;7:491-499.

38. Meyer BJ, Larkin TA, Owen AJ, et al. Limited lipidlowering effects of regular consumption of whole soybean foods. Ann Nutr Metab 2004;48:67-78.

39. Cheong SH, Furuhashi K, Ito K, et al. Antihyperglycemic effect of equol, a daidzein derivative, in cultured L6 myocytes and ob/ob mice. Mol Nutr Food Res 2014;58: 267-277.

40. Khosla S, Riggs B. Pathophysiology of age-related bone loss and osteoporosis. Endocrinol Metab Clin N Am 2005; 34:1015-1030.

41. Ishimi Y. Dietary equol and bone metabolism in postmenopausal Japanese women and osteoporotic mice. J Nutr 2010;140:1373S-1376S.
42. Weaver C, Legette L. Equol, via dietary sources or intestinal production, may ameliorate estrogen deficiencyinduced bone loss. J Nutr 2010;140:1377S-1379S.

43. Taku K, Melby MK, Kurzer MS, et al. Effects of soy isoflavone supplements on bone turnover markers in menopausal women: Systematic review and meta-analysis of randomized controlled trials. Bone 2010;47:413-423.

44. Ohtomo T, Uehara M, Peñalvo JL, et al. Comparative activities of daidzein metabolites, equol and O-desmethylangolensin, on bone mineral density and lipid metabolism in ovariectomized mice and in osteoclast cell cultures. Eur J Nutr 2008;47:273279.

45. Shimada T, Kakitani M, Yamazaki Y, et al. Targeted ablation of Fgf23 demonstrates an essential physiological role of FGF23 in phosphate and vitamin D metabolism. J Clin Invest 2004;113:561-568.

46. Carrillo-López N, Román-García P, Rodríguez-Rebollar A, et al. Indirect Regulation of PTH by Estrogens May Require FGF23. J Am Soc Nephrol 2009;20:2009-2017.

47. Uemura $\mathrm{H}$, Irahara $\mathrm{M}$, Yoneda $\mathrm{N}$, et al. Close correlation between estrogen treatment and renal phosphate reabsorption capacity. J Clin Endocrinol Metab 2000;85:1215-1219.

48. Davinelli S, Scapagnini G, Marzatico F, et al. Influence of equol and resveratrol supplementation on health-related quality of life in menopausal women: A randomized, placebo-controlled study. Maturitas 2017;96:77-83.

49. Kruger MC, Middlemiss C, Katsumata S, et al. The effects of green kiwifruit combined with isoflavones on equol production, bone turnover and gut microflora in healthy postmenopausal women. Asia Pac J Clin Nutr 2018;27:347.

Address correspondence to: Khin Zay Yar Myint, MBBS, MHS Tokyo Midtown Medical Center 9-7-1, Akasaka Minato-ku 107-6206 Japan

E-mail: khinzayarmyint@gmail.com 